\title{
On Throughput of Compressive Random Access for One Short Message Delivery in IoT
}

\author{
Jinho Choi
}

\begin{abstract}
In this paper, we study compressive random access (CRA) with two stages for machine-type communication (MTC) in cellular Internet-of-Things (IoT). In particular, we consider the case that each user (IoT device or sensor) has only one short message (of the same length) when it is activated to send data in IoT applications. Two different CRA-based random access schemes are discussed (one is conventional and the other is new based on a simplified handshaking process). Based on the throughput analysis, we show that the CRA-based random access scheme with simplified handshaking process can outperform as its length of payload is adaptively decided depending on the number of active users. Simulation results confirm that the derived throughput expressions agree with them and can be used to design a random access system for MTC with each active device or sensor that has one short message.
\end{abstract}

Index Terms-Machine-Type Communication; Compressive Random Access; the Internet-of-Things

\section{INTRODUCTION}

Recently, the Internet of Things (IoT) has been extensively studied and a number of different applications of the IoT are considered for smart cities and factories [1] [2]. To support the connectivity for various IoT applications, cellular IoT has been considered. For example, in [3], a deployment study of narrowband IoT (NB-IoT) [4] is carried out to support IoT applications over a large area. In cellular IoT, machine-type communication (MTC) [5] [4] plays a crucial role in providing the connectivity for IoT devices and sensors [6]. Due to sparse activity of those devices and sensors, MTC is usually based on random access to keep signaling overhead low [5] [4] [7] [8] [9].

Due to a large number of IoT devices and sensors, it is expected to support massive connectivity in MTC [10]. To this end, the notion of massive multiple input multiple output (MIMO) [11] can be considered. In [12] [13] [14] [15], massive MIMO based random access schemes are studied for massive MTC, where a base station (BS) is equipped with a large number of antenna elements. In [16] [17], massive MIMO is used to collect data from sensors in wireless sensor networks (WSNs). Based on [18], it seems that massive MIMO is a solution to massive MTC, as the capacity becomes unbounded in the presence of pilot contamination, which may allow to support a very large number of devices in each cell.

However, if a large number of antenna elements at a BS are not available (due to various reasons including cost), it may be necessary for BS with single antenna to consider other approaches that have a high spectral efficiency or throughput

The author is with the School of Information Technology, Deakin University, Geelong, VIC 3220, Australia (e-mail: jinho.choi@ deakin.edu.au). This research was supported by the Australian Government through the Australian Research Council's Discovery Projects funding scheme (DP200100391). to support MTC. In order to improve the throughput of random access for MTC, the notion of compressive sensing has been applied to multiuser detection (MUD) at a BS (equipped with single antenna) in [19] [20]. In [21] [22] [23], in order to estimate the channel state information (CSI) for coherent detection, two-stage approaches are considered for random access, where in general the first stage is to transmit preambles or pilots (so that the BS can estimate the CSI of active users) and the following second stage is to transmit data packets. The resulting approach is called compressive random access (CRA) [24], as the notion of compressive sensing [25] [26] can be employed to exploit the sparse activity of users in MUD. In general, CRA can be seen as a multichannel random access scheme, where each (multiple access) channel can be characterized by a preamble in the first stage. In the second stage, the preamble can be used as a spreading sequence for MUD, which results in code division multiple access (CDMA) based random access in [19] [20]. As shown in [23], it is possible to use different spreading code (but associated with the preamble in the first stage) for spreading in the second stage.

The notion of CRA is applied to an existing random access scheme for MTC in [27]. As stated in [27] [28], a salient feature of CRA is that the preambles can be non-orthogonal. If orthogonal preambles are used, the number of preambles becomes the length of preambles. However, if non-orthogonal preambles are used, the number of preambles can be much larger than the length of preambles, which can effectively reduce the probability of preamble collision (PC) and improve the performance of random access.

In this paper, we study two two-stage random access schemes based on CRA, where the first stage is used to transmit preambles by active users so that the BS is able to estimate CSI of active users, while the second stage is used to transmit data packets. Throughout the paper, a special case is considered, where each active user has only one data packet of the same length. This might be the case where each active user has a short message (e.g., a few tens bytes) in IoT applications [29], where a user is an IoT device or sensor. In general, since the length of data packet is short, it is desirable to have a low signaling overhead (which justifies the use of random access) as well as a high throughput. We show that CRA-based twostage random access schemes can have high throughput (than that of conventional multichannel ALOHA). One of the two CRA-based schemes in this paper is similar to conventional grant-free CRA-based schemes in [22] [23] (as no access grant is pursued by users). While the other scheme differs from the conventional schemes, it can be seen as a special case of [27] with a simplified handshaking process. Since we only 
consider one short packet transmission from each active user, a specific implementation of the second stage becomes possible as will be explained later in this paper. The resulting scheme can provide a higher throughput than conventional grant-free CRA-based scheme. In summary, the main contribution of the paper is two-fold: $i$ ) a new CRA-based random access scheme with a simplified handshaking process is proposed, which might be suitable for IoT applications where each device or sensor has only one short message to send in each access; ii) a throughput analysis is studied with the length of the second stage that varies and depends on the number of active users.

Note that the proposed approach in this paper differs from that in [23] as the feedback from the BS is exploited (through a simplified handshaking process) and that in [27] as the number of slots for data packet transmissions is adaptively decided to improve the throughput.

The rest of the paper is organized as follows. In Section II, we present the system model for two CRA-based random access schemes. The throughput of two CRA-based random access schemes is analyzed in Section III. In Section IV, we briefly discuss some other issues that are not studied in Section III. Simulation results are presented in Section V, and the paper is concluded with some remarks in Section VI.

Notation: Matrices and vectors are denoted by upper- and lower-case boldface letters, respectively. The superscripts $\mathrm{T}$ and $\mathrm{H}$ denote the transpose and complex conjugate, respectively. The support of a vector is denoted by $\operatorname{supp}(\mathbf{x})$ (which is the number of the non-zero elements of $\mathbf{x}) . \mathbb{E}[\cdot]$ and $\operatorname{Var}(\cdot)$ denote the statistical expectation and variance, respectively. $\mathcal{C N}(\mathbf{a}, \mathbf{R})$ represents the distribution of circularly symmetric complex Gaussian (CSCG) random vectors with mean vector a and covariance matrix $\mathbf{R}$.

\section{SySTEM MODEL}

Suppose that a random access system consists of a BS and a number of users, where all the users are synchronized. In this paper, we consider a particular case where each active user has only one packet to send. For example, in IoT applications with a number of environmental sensors, each sensor may have a fixed small number of bytes to send to the BS. In this case, a complicated approach based on handshaking (e.g., [4] [5]) might result in unnecessary signaling overhead. To avoid it, simple approaches based on two stages can be considered with a pool of pre-determined preambles that are non-orthogonal sequences. In this paper, we consider two slightly different approaches with two stages.

\section{A. CRA-1}

In this subsection, we consider a random access approach with two stages. Similar to [23] in Stage 1, an active user is to randomly choose a preamble from the pool. Let $\boldsymbol{\psi}_{l}$ be the $l$ th preamble in the pool of $L$ preambles, $\left\{\boldsymbol{\psi}_{1}, \ldots, \boldsymbol{\psi}_{L}\right\}$, where $L$ represents the number of available preambles. Here, $\psi_{l}$ is a sequence of length $N$. The BS receives the preambles transmitted by active users and can detect them using a CSbased approach.
For convenience, denote by $T_{\mathrm{P}}=N T_{\mathrm{s}}$ the duration of Stage 1 , where $T_{\mathrm{s}}$ is the unit symbol duration or the inverse of the system bandwidth (with an ideal pulse shaping filter). For convenience, $T_{\mathrm{s}}$ is normalized, i.e., $T_{\mathrm{s}}=1$ (as a result, $T_{\mathrm{P}}=N$ ). We assume that $L \gg N$ (since the preambles are non-orthogonal, there can be more than $N$ sequences). The resulting scheme is referred to as CRA [24] in this paper. In CRA, it is usually assumed that the number of active users, denoted by $K$, is sufficiently small, i.e., $K \ll N$. In Stage 1 , the BS is able to estimate the channel coefficients of $K$ active users in conjunction with the detection of transmitted preambles using CS algorithms. In this paper, we assume that the bandwidth is sufficiently narrow so that the channels are modeled as flat fading channels as in [20] (note that frequencyselective fading channels are considered in [21] [24]).

In Stage 2, each active user transmits its data packet of $M$ symbol with spreading by the preamble that is used in Stage 1 (similar to CDMA as in [19] [20]). Thus, the spread packet duration becomes $N T_{\mathrm{D}}$, where $T_{\mathrm{D}}=M T_{\mathrm{s}}=M$ denotes the packet duration (in unit time). Since the transmitted preambles can be detected in Stage 1, they can be used for MUD [30] in Stage 2 to recover data symbols from spread signals. Note that if $K \leq N$, where $N$ is regarded as the spreading gain, MUD can recover data symbols from $K$ active users (provided that the signal-to-noise ratio (SNR) is sufficiently high as well as the correlation between preambles is sufficiently low).

For convenience, the resulting approach is referred to as CRA-1. In Fig. 1, we illustrate a session consisting of Stage 1 for preamble transmissions and Stage 2 for data packet transmissions. Note that at the end of a session, there is a feedback from the BS to users to inform the success or failure of data packet decoding.

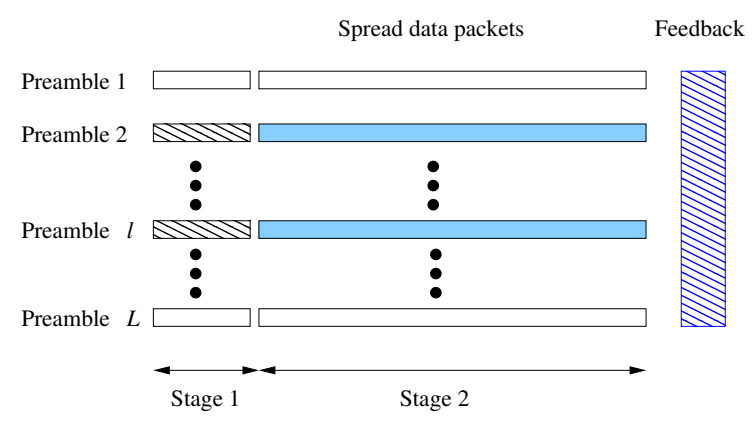

Fig. 1. CRA-1 with 2 stages, where active users' preambles are denoted by shaded blocks and their data packets are shown in light blue.

Let $h_{k}$ and $l(k)$ denote the channel coefficient from active user $k$ to the BS and the preamble index chosen by active user $k$, respectively. Since there are $K$ active users, the received signal at the BS during Stage 1 is given by

$$
\mathbf{y}=\sum_{k=1}^{K} \boldsymbol{\psi}_{l(k)} \sqrt{P_{k}} h_{k}+\mathbf{n}=\Psi_{\mathbf{s}}+\mathbf{n}
$$

where $\boldsymbol{\Psi}=\left[\begin{array}{lll}\boldsymbol{\psi}_{1} & \ldots & \boldsymbol{\psi}_{L}\end{array}\right] \in \mathbb{C}^{N \times L}, \mathbf{s}$ is a $K$-sparse vector, $P_{k}$ is the transmit power of active user $k$, and $\mathbf{n} \sim \mathcal{C N}\left(\mathbf{0}, N_{0} \mathbf{I}\right)$ 
is the background noise vector. The received signal at the BS during Stage 2 is given by

$$
\begin{aligned}
\mathbf{r}_{m} & =\sum_{k=1}^{K} \boldsymbol{\psi}_{l(k)} \sqrt{P_{k}} h_{k} d_{k, m}+\mathbf{n}_{m} \\
& =\boldsymbol{\Psi} \mathbf{s}_{m}+\mathbf{n}_{m}, m=0, \ldots, M-1,
\end{aligned}
$$

where $d_{k, m}$ is the $m$ th data symbol of data packet from active user $k$ and $\mathbf{n}_{m} \sim \mathcal{C N}\left(\mathbf{0}, N_{0} \mathbf{I}\right)$ is the background noise vector. Here, $\mathbf{s}_{m}$ is a $K$-sparse vector that has the same support as $\mathbf{s}$ in (1). Thus, the received signals during Stage 1 and Stage 2 can be seen as $\left[\begin{array}{llll}\mathbf{y}^{\mathrm{T}} \mathbf{r}_{0}^{\mathrm{T}} & \ldots & \mathbf{r}_{M-1}^{\mathrm{T}}\end{array}\right]^{\mathrm{T}}$, which is a vector of length $N+M N=(1+M) N$.

In fact, $\left\{\mathbf{y}, \mathbf{r}_{0}, \ldots, \mathbf{r}_{M-1}\right\}$ can be seen as multiple measurement vectors (MMV) in the context of compressive sensing [31] [32]. It is known that a sufficient and necessary condition to estimate the support of $\mathbf{s}$ or the indices of the transmitted preambles is given by

$$
K<\frac{\operatorname{spark}(\mathbf{\Psi})-1+\operatorname{rank}(\mathbf{S})}{2},
$$

where $\mathbf{S}=\left[\begin{array}{llll}\mathbf{s} & \mathbf{s}_{0} & \ldots & \mathbf{s}_{M-1}\end{array}\right]$ and $\operatorname{spark}(\mathbf{\Psi})$ is the smallest number of columns from $\Psi$ that are linearly dependent [33]. For a random vector for $\boldsymbol{\Psi}, \operatorname{spark}(\boldsymbol{\Psi})-1=\operatorname{rank}(\boldsymbol{\Psi})=N$ w.p. 1 [34]. Thus, if $\operatorname{rank}(\mathbf{S})=N$, from (3), it is possible to detect up to $N-1$ transmitted preambles. Then, with the detected transmitted preambles, the channel coefficients can be estimated from $\mathbf{y}$. With the estimated channel coefficients as well as detected transmitted preambles, data packets can be decoded from $\left\{\mathbf{r}_{0}, \ldots, \mathbf{r}_{M-1}\right\}$.

In [28], the performance of CRA is compared with that of multichannel ALOHA. In multichannel ALOHA, it is assumed that the preambles are orthogonal and each preamble represents an orthogonal (multiple access) channel. As a result, the number of preambles is equal to the length of preamble, i.e., $L=N$. In this case, it is not necessary to use CS algorithms, because each channel is seen as an independent channel and a simple correlator becomes optimal to detect the presence of signal in each channel (i.e., no CS-based MUD is required). However, since the number of preambles in CRA can be higher than that in multichannel ALOHA, it is shown that the throughout of CRA can be higher ${ }^{1}$ than that of multichannel ALOHA by a factor of 2 [28].

CRA-1 is often considered to be a grant-free approach [23], because an active user can transmit a data packet without asking a dedicated channel (for payload).

\section{B. $C R A-2$}

In this subsection, we consider a slightly different approach from CRA-1, while its Stage 1 is the same as that in CRA-1.

After Stage 1 (i.e., once the BS is able to detect the transmitted preambles), suppose that the BS sends feedback signal to users to inform the indices of detected transmitted preambles, which is referred to as Feedback 1. Let $D$ denote the number of the detected preambles and denote by $l_{d} \in\{1, \ldots, L\}$

\footnotetext{
${ }^{1}$ This is due to the fact that the increase of preambles reduces the probability of PC.
}

the index of the $d$ th detected preamble with the following increasing order: $l_{1}<l_{2}<\ldots<l_{D}$. In Stage 2, there are $D$ slots for transmissions of data packets. The BS expects to receive a data packet during the $d$ th slot from the active user transmitting the preamble of index $l_{d}$. As a result, the duration of Stage 2 is $D T_{\mathrm{D}}=D M$ as illustrated in Fig. 2. Note that since $D$ varies, the length of Stage 2 varies from a session to another. The length of session is the sum of the lengths of Stages 1 and 2 and Feedbacks 1 and 2.

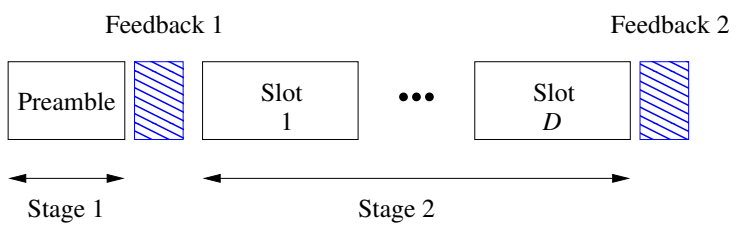

Fig. 2. CRA-2 with 2 stages (when each active user has only one data packet to send), where the number of slots for uplink data packets from active users, $D$, varies.

Since an active user that transmits a preamble, say $l_{d}$, knows that the slot for its data packet is the $d$ th slot from Feedback 1, it can transmit a data packet in the $d$ th slot in Stage 2. At the end of Stage 2, the BS sends another feedback signal to the users to inform success or failure of decoding of data packets, which is referred to as Feedback 2. The resulting scheme is referred to as CRA-2.

It is noteworthy that CRA-2 can be seen as a special case of the approach in [27], where CRA is used only for connection establishment. Thanks to the presence of Feedback 1 , the operation of Stage 1 in CRA-2 is seen as a simplified handshaking process as in [27]. Furthermore, since only one data packet per active user is assumed in this paper, in Stage 2 , channels for payloads (or data packets) can be easily reserved without further requests from active users (which is not considered in [27]). Note that if each active user has a different number of data packets to transmit, the length of slot can be set to the maximum length of data packet in CRA-2 at the cost of degraded spectral efficiency.

For simplicity, we assume that the duration of Feedback 1 is equal to that of Feedback 2 and the total duration of feedback is denoted by $\tau$. Note that in CRA-1, there might be feedback to inform whether or not data packets are successfully decoded. In this case, CRA-1 has only Feedback 2. In Table I, we summarize the duration of each stage and feedback in CRA-1 and CRA-2. Throughout the paper, we assume that $\tau \ll T_{\mathrm{D}}$. A notable difference between CRA-1 and CRA-2 is that the length of Stage 2 of CRA-2 is varying, but that of CRA-1 is fixed. From this, it is expected that CRA2 can perform better than CRA-1 when the number of active users, $K$, is less than the spreading gain or the length of preambles, $N$.

Note that CRA-1 requires MUD [30] [35] to detect multiple spread signals simultaneously. On the other hand in CRA-2, since each slot has only one data packet, a conventional singleuser detector can be used. From this, in terms of data packet detection/decoding, CRA-2 could be easier to implement than CRA-1. 
TABLE I

THE DURATION OF EACH STAGE AND FEEDBACK IN CRA-1 AND CRA-2.

\begin{tabular}{|c|c|c|}
\hline & CRA-1 & CRA-2 \\
\hline \hline Duration of Stage 1 & $T_{\mathrm{P}}=N$ & $T_{\mathrm{P}}=N$ \\
\hline Duration of Stage 2 & $N T_{\mathrm{D}}=N M$ (fixed) & $D T_{\mathrm{D}}=D M$ (varying) \\
\hline Duration of Feedback & $\frac{\tau}{2}$ & $\tau$ \\
\hline
\end{tabular}

\section{Throughrut ANALYsis}

In this section, we consider the throughput of the two-stage random access schemes, i.e., CRA-1 and CRA-2. We first identify possible error events and find the throughput.

\section{A. Error Events}

We assume that there is no feedback error from the BS to users (i.e., Feedbacks 1 and 2 are always successful).

In Stage 1 (for both CRA-1 and CRA-2), there are the following error events at the BS:

- False alarm (FA): The BS erroneously detects a preamble that is not transmitted by any active user.

- Missed detection (MD): The BS cannot detect a preamble transmitted by an active user.

- PC: A detected preamble is transmitted by multiple active users.

Note that if each user has a unique preamble (as a signature sequence), there is no preamble collision. However, if the number of users is large, it is difficult to manage a set of signature sequences. Furthermore, if there are new or outgoing users with unique sequences, the BS needs to update the set of signature sequences. Therefore, it is convenient to have a pool of pre-determined preambles that are shared by users at the cost of preamble collision.

\section{B. Analysis of CRA-2 with Packet Loss}

Since the analysis of CRA-1 is straightforward once we analyze CRA-2, we first consider CRA-2 in this subsection. Throughout this section, we assume that the active users associated with MD and PC events drop their packets.

In Stage 2 of CRA-2, for tractable analysis, we assume that all the transmitted data packets are successfully decodable if there is no collision. In Stage 1, when there are multiple active users that transmit the same preamble, the BS detects the preamble and broadcasts its index through Feedback 1. Then, all the active users that choose the preamble transmit their data packets in Stage 2, which results in packet collision. Thus, the packet collision (in Stage 2) is directly related to preamble collision (in Stage 1).

For convenience, define

$$
X_{k, l}= \begin{cases}1, & \text { if active user } k \text { chooses preamble } l \\ 0, & \text { o.w. }\end{cases}
$$

In addition, let

$$
\mathcal{B}=\left\{l: \sum_{k=1}^{K} X_{k, l} \geq 1\right\} \text { and } \mathcal{B}_{1}=\left\{l: \sum_{k=1}^{K} X_{k, l}=1\right\} \text {. }
$$

Clearly, $\mathcal{B}_{1} \subseteq \mathcal{B}$. Let $B=|\mathcal{B}|$ and $B_{1}=\mathcal{B}_{1}$. Then, $B$ becomes the number of the selected preambles by $K$ active users, while $B_{1}$ becomes the number of the preambles, each of which is selected by only one active user. Thus, $B \geq B_{1}$. Let $\mathcal{B}_{2}=$ $\mathcal{B} \backslash \mathcal{B}_{1}$ and $B_{2}=\left|\mathcal{B}_{2}\right|$. Clearly, $B_{2}=B-B_{1}$, which becomes the number of the preambles, each of which is selected by multiple active users.

In Fig. 3, an example is illustrated with $K=3$ active users and $L=4$ preambles. Here, $X_{1,4}=X_{2,2}=X_{3,2}=1$, while all the other $X_{k, l}$ 's are zero. Clearly, $\mathcal{B}=\{2,4\}$ and $\mathcal{B}_{1}=\{4\}$.

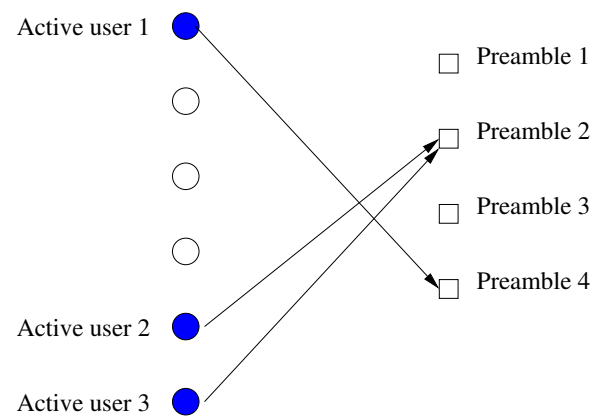

Fig. 3. There are $K=3$ active users and $L=4$ preambles, where active user 1 chooses preamble 4 and active users 2 and 3 choose preamble 2 .

At the BS, if FA and MD events do not happen, i.e., the BS is able to detect all transmitted preambles, $D$ becomes $B$. However, due to FA and MD events, $D$ could be different from $B$. Consider FA events. The number of erroneously detected preambles by FA events is denoted by $D_{3}$. Clearly, $D_{3}$ cannot be greater than $L-B$. Denote by $D_{1}$ and $D_{2}$ the numbers of correctly detected preambles among those in $\mathcal{B}_{1}$ and $\mathcal{B}_{2}$, respectively. Then, we have

$$
D=D_{1}+D_{2}+D_{3} \text {. }
$$

Among $D$ slots, $D_{3}$ slots will not have any data packets as there are no active users associated with them (due to FA events). In addition, each of $D_{2}$ slots may have collided packets as there are multiple active users associated with it (due to PC events). Consequently, there are only $D_{1}$ slots, where each slot has only one data packet from an active user (i.e., without collision).

Let

$$
\begin{aligned}
U_{l} & =\mathbb{1}\left(\sum_{k=1}^{K} X_{k, l}=1\right) \in\{0,1\} \\
W_{l} & =\mathbb{1}\left(\sum_{k=1}^{K} X_{k, l} \geq 2\right) \in\{0,1\},
\end{aligned}
$$

where $\mathbb{1}(\cdot)$ represents the indicator function. If we assume that each active user randomly chooses one of $L$ preambles, it can be shown that

$$
\operatorname{Pr}\left(U_{l}=1\right)=\left(\begin{array}{c}
K \\
1
\end{array}\right) \frac{1}{L}\left(1-\frac{1}{L}\right)^{K-1}=\frac{K}{L}\left(1-\frac{1}{L}\right)^{K-1} .
$$


In addition, we have

$$
\operatorname{Pr}\left(W_{l}=1\right)=1-\frac{K}{L}\left(1-\frac{1}{L}\right)^{K-1}-\left(1-\frac{1}{L}\right)^{K} .
$$

For convenience, let $\alpha_{1}=\frac{K}{L}\left(1-\frac{1}{L}\right)^{K-1}$ and $\alpha_{2}=$ $\left(1-\frac{1}{L}\right)^{K}$. Then, $\operatorname{Pr}\left(U_{l}=1\right)=\alpha_{1}$ and $\operatorname{Pr}\left(W_{l}=1\right)=$ $1-\alpha_{1}-\alpha_{2}$.

For tractable analysis, we consider the following assumption.

A1) The $U_{l}$ 's are independent of each other. In addition, the $W_{l}$ 's are independent of each other.

The above independent assumption is not valid, but would be a good approximation for a sufficiently large $L$ with $K \ll L$. In addition, for convenience, let

$$
\bar{D}_{i}(K)=\mathbb{E}\left[D_{i} \mid K\right], \quad i=1,2,3 .
$$

Lemma 1: Suppose that the events of FA and MD are independent and the probabilities of MD (per transmitted preamble) and FA (per untransmitted preamble) are denoted by $P_{\mathrm{MD}}$ and $P_{\mathrm{FA}}$, respectively. Under the assumption of $\mathbf{A 1}$, we have

$$
\begin{aligned}
& \bar{D}_{1}(K)=\left(1-P_{\mathrm{MD}}\right) L \alpha_{1} \\
& \bar{D}_{2}(K)=\left(1-P_{\mathrm{MD}}\right) L\left(1-\alpha_{1}-\alpha_{2}\right) \\
& \bar{D}_{3}(K)=P_{\mathrm{FA}} L \alpha_{2} .
\end{aligned}
$$

Proof: See Appendix A.

We now consider a steady state analysis using (10) under the following assumption.

A2) The number of active users, $K$, follows a Poisson distribution with a traffic intensity $\lambda$ (in the number of users per unit time).

Let $D(t)$ denote the number of detected preambles in session $t$ and denote by $Y(t)$ the length of session $t$. Then, we have

$$
Y(t)=T_{\mathrm{P}}+\tau+T_{\mathrm{D}} D(t),
$$

where $\tau$ represents the total length of Feedbacks 1 or 2 . For convenience, let $\tilde{T}_{\mathrm{P}}=T_{\mathrm{P}}+\tau$. Then, the number of the active users in slot $t+1$ follows the following distribution:

$$
\operatorname{Pr}(K(t+1)=k)=\frac{(\lambda Y(t))^{k}}{k !} e^{-\lambda Y(t)} .
$$

We assume that the mean of $Y(t)$ exists and is denoted by $\bar{Y}=\mathbb{E}[Y(t)]$ and let $\beta_{2}=\lambda \bar{Y}$. Furthermore, in the steady state $(t \rightarrow \infty)$, we assume that $K$ has the following steadystate distribution:

$$
\operatorname{Pr}(K=k)=\frac{\beta_{2}^{k}}{k !} e^{-\beta_{2}} .
$$

In the following result, we obtain an expression for $\beta_{2}$ if $\bar{Y}$ exists.

Lemma 2: With the steady-state distribution of $K$ in (13), $\beta_{2}$ is given by

$$
\begin{aligned}
& \beta_{2}=L\left(c_{1}+\mathbb{W}\left(-c_{2} e^{-c_{1}}\right)\right) \\
& \bar{D}=L\left(1-P_{\mathrm{MD}}+\frac{\mathbb{W}\left(-c_{2} e^{-c_{1}}\right)}{\lambda T_{\mathrm{D}}}\right) .
\end{aligned}
$$

where $\mathbb{W}(\cdot)$ denotes the Lambert $\mathrm{W}$ function ${ }^{2}$ and

$$
\begin{aligned}
& c_{1}=\lambda\left(\frac{\tilde{T}_{\mathrm{P}}}{L}+T_{\mathrm{D}}\left(1-P_{\mathrm{MD}}\right)\right) \\
& c_{2}=\lambda T_{\mathrm{D}}\left(1-P_{\mathrm{MD}}-P_{\mathrm{FA}}\right) .
\end{aligned}
$$

Proof: See Appendix B.

We can also obtain the mean of $D_{1}$ as follows:

$$
\begin{aligned}
\bar{D}_{1} & =\mathbb{E}\left[D_{1}(K)\right]=\sum_{k=1}^{\infty} \bar{D}_{1}(k) \frac{\beta_{2}^{k}}{k !} e^{-\beta_{2}} \\
& =\left(1-P_{\mathrm{MD}}\right) \beta_{2} e^{-\frac{\beta_{2}}{L}} .
\end{aligned}
$$

Thus, the average number of successfully transmitted packets per session (or the ratio of the average number of successfully transmitted packets to the average session length) is given by

$$
\eta_{2}=\frac{\bar{D}_{1}}{\bar{Y}}=\frac{\left(1-P_{\mathrm{MD}}\right) \beta_{2} e^{-\frac{\beta_{2}}{L}}}{\tilde{T}_{1}+\bar{D} T_{2}}=\lambda\left(1-P_{\mathrm{MD}}\right) e^{-\frac{\beta_{2}}{L}},
$$

which is the throughput of CRA-2.

Note that we have assumed that the error probabilities, $P_{\mathrm{MD}}$ and $P_{\mathrm{FA}}$, are constants (i.e., independent of $K$ ). This assumption will be discussed in Subsection IV-B.

\section{Analysis of CRA-1 with Packet Loss}

In this subsection, we focus on the throughput of CRA-1.

Let $Z=T_{\mathrm{P}}+\frac{\tau}{2}+N T_{\mathrm{D}}$. Then, the probability that $K=k$ in CRA-1 is given by $\operatorname{Pr}(K=k)=\frac{\eta_{1}^{k}}{k !} e^{-\beta_{1}}$, where $\beta_{1}=\lambda Z$, which is seen as the average number of active users. In CRA-1, as mentioned earlier, we assume that if $K \geq N$ (i.e., the number of active users is greater than the spreading gain), MUD fails. Thus, the average number of successfully recovered packets becomes

$$
\begin{aligned}
\bar{K}_{1} & =\sum_{k=1}^{N-1} \bar{D}_{1}(k) \operatorname{Pr}(K=k) \\
& =\left(1-P_{\mathrm{MD}}\right) \beta_{1} e^{-\beta_{1}} \sum_{k=0}^{N-2} \frac{\left(\beta_{1}\left(1-\frac{1}{L}\right)\right)^{k}}{k !} \\
& =\left(1-P_{\mathrm{MD}}\right) \beta_{1} e^{-\beta_{1}} \frac{\Gamma\left(N-1, \beta_{1}\left(1-\frac{1}{L}\right)\right)}{(N-1) !},
\end{aligned}
$$

where $\Gamma(n, x)=\int_{x}^{\infty} t^{n-1} e^{-t} d t$ represents the upper incomplete gamma function. Then, the throughput of CRA-1 (as the ratio of the average number of successfully transmitted packets to the session length) is given by

$$
\eta_{1}=\frac{\bar{K}_{1}}{Z}=\lambda\left(1-P_{\mathrm{MD}}\right) e^{-\beta_{1}} \frac{\Gamma\left(N-1, \beta_{1}\left(1-\frac{1}{L}\right)\right)}{(N-1) !} .
$$

For convenience, let $V$ be a Poisson random variable with mean $\beta_{1}\left(1-\frac{1}{L}\right)$. Then, it can be shown that

$$
\eta_{1}=\lambda\left(1-P_{\mathrm{MD}}\right) e^{-\frac{\beta_{1}}{L}} \operatorname{Pr}(V \leq N-2) .
$$

From (18) and (21), we can see that if $\beta_{1} \approx \beta_{2}$ (which is the case that the average duration of Stage 2 in CRA-2 is

\footnotetext{
${ }^{2}$ The Lambert function is the inverse function of $f(x)=x e^{x}$, i.e., $x=$ $\mathbb{W}(y)$, where $y=x e^{x}$.
} 
equal to that in CRA-1 or $\bar{D} \approx N), \eta_{2}$ might be larger than $\eta_{1}$ as $\operatorname{Pr}(V \leq N-2) \leq 1$ and the difference may increase if $\mathbb{E}[V]-(N-2)=\beta_{1}\left(1-\frac{1}{L}\right)-(N-2)$ is positive and increases.

Note that when multichannel ALOHA is used, in (19), $L$ is to be replaced with $N$. In addition, since all the channels are orthogonal, the BS can decode up to $N$ packets when each packet is transmitted with a different preamble. Thus, the throughput of multichannel ALOHA becomes

$$
\eta_{\mathrm{ma}}=\lambda\left(1-P_{\mathrm{MD}}\right) e^{-\beta_{1}} \frac{\Gamma\left(N, \beta_{1}\left(1-\frac{1}{N}\right)\right)}{N !} .
$$

For a sufficiently large $N$, we can see that $\eta_{1}>\eta_{\text {ma }}$ for $L>N$.

\section{OTHER ISSUES}

In this section, we discuss two key issues that are not considered for the throughput analysis in Section III.

\section{A. Stability Issue with Re-Transmissions}

In Section III, we do not consider re-transmissions of unsuccessful data packets. Since there are multiple preambles, an active user with unsuccessful data packet can immediately transmit another randomly chosen preamble in the next session, which can be seen as fast retrial [36]. For CRA-1, fast retrial was considered in [28] with its stability analysis when the access probability is controlled. In this subsection, we briefly discuss stability issues with fast retrial for CRA-2.

Let $t$ be the index for sessions. Thus, $D_{i}(t)$ represents $D_{i}$ in session $t$. After session $t$, the number of the backlogged users due to preamble collision and MD events is given by

$$
Z(t)=K(t)-D_{1}(t)
$$

where $K(t)$ denotes the number of users at session $t$. Thus, if the active users with unsuccessfully transmitted packets attempt to transmit preambles in the next session based on fast retrial, we have

$$
\begin{aligned}
\mathbb{E}[K(t+1) \mid D(t)] & =\lambda Y(t)+Z(t) \\
& =\lambda\left(T_{\mathrm{P}}+\tau+T_{\mathrm{D}} D(t)\right)+Z(t) .
\end{aligned}
$$

Let $\mathbb{E}[D(t) \mid K(t)]=\sum_{i=1}^{3} \bar{D}_{i}(K(t))$. Since

$$
\begin{aligned}
\mathbb{E}[K(t+1) \mid K(t)] & =\lambda\left(\tilde{T}_{\mathrm{P}}+T_{\mathrm{D}} \mathbb{E}[D(t) \mid K(t)]\right) \\
& +K(t)-\mathbb{E}\left[D_{1}(t) \mid K(t)\right] \\
& =\lambda\left(\tilde{T}_{\mathrm{P}}+T_{\mathrm{D}}\left(\sum_{i=2}^{3} \bar{D}_{i}(K(t))\right)\right) \\
& +K(t)-\left(1-\lambda T_{\mathrm{D}}\right) \mathbb{E}\left[D_{1}(t) \mid K(t)\right],(25)
\end{aligned}
$$

the drift [37] becomes

$$
\begin{aligned}
\delta(K)= & \mathbb{E}[K(t+1) \mid K(t)=K]-K \\
= & \lambda\left(\tilde{T}_{\mathrm{P}}+T_{\mathrm{D}}\left(\sum_{i=2}^{3} \bar{D}_{i}(K)\right)\right) \\
& -\left(1-\lambda T_{\mathrm{D}}\right) \bar{D}_{1}(K) .
\end{aligned}
$$

From (10), it can be shown that $\lim _{K \rightarrow \infty} \bar{D}_{1}(K)=$ $\lim _{K \rightarrow \infty} \bar{D}_{3}(K)=0$ and $\lim _{K \rightarrow \infty} \bar{D}_{2}(K)=\left(1-P_{\mathrm{MD}}\right) L$. Then, it can be shown that $\delta(K)>0$ for $K \geq K_{0}$, where $K_{0}$ is finite. This implies that CRA-2 becomes unstable if $K$ is sufficiently large, which results from a number of unsuccessful packets that are to be re-transmitted. To avoid this problem, unsuccessful packets can be dropped as in Section III or access control schemes can be considered, which might be studied in the future.

\section{B. Error Probabilities}

In CRA, for MUD, greedy CS algorithms [38] are used to detect all active users (actually their preambles) under the assumption that the number of active users is sufficiently small, e.g., [20] [21]. In [27], LASSO [39] is applied to MUD in order to detect transmitted preambles. In general, the detection performance of transmitted preambles depends on the algorithm used for MUD. In other words, $P_{\mathrm{MD}}$ and $P_{\text {FA }}$ depend on the algorithm used. Thus, in this subsection, in order to avoid the performance dependency on a particular algorithm, we mainly focus on optimal MUD and consider $P_{\mathrm{MD}}$ and $P_{\mathrm{FA}}$ based on the maximum likelihood $(\mathrm{ML})^{3}$ criterion.

Provided that there are $K$ active users, it might be unlikely to detect more than $K+1$ users in the event of FA or less than $K-1$ users in the event of MD. Thus, for tractable analysis, we only consider the case that the BS detects $K+1$ transmitted preambles for FA events. Likewise, for MD events, the case that the BS detects $K-1$ transmitted preambles is studied. In addition, for simplicity, we assume that there is no preamble collision. Thus, $B=K$ in this subsection.

It is assumed that all the preambles are normalized as $\left\|\boldsymbol{\psi}_{l}\right\|_{2}=1$. Let the support set of $\mathbf{s}$ be

$$
\mathcal{S}=\operatorname{supp}(\mathbf{s})=\{l(1), \ldots, l(K)\} .
$$

In addition, for MD events, define a vector $\mathbf{s}_{k}^{\prime}$ as follows:

$$
\left[\mathbf{s}-\mathbf{s}_{k}^{\prime}\right]_{l}= \begin{cases}\sqrt{P_{k}} h_{k}, & \text { if } l=l(k) \\ 0, & \text { o.w. }\end{cases}
$$

Clearly, $\mathbf{s}_{k}^{\prime}$ is a $(K-1)$-sparse vector that does not include the signal from the $k$ th active user. From (1), if the ML detector is used with known CSI (i.e., all users' channel coefficients, $h_{k}$ 's), it can choose $\mathbf{s}_{k}^{\prime}$ with the following probability:

$$
\begin{aligned}
P_{k}^{\prime} & =\operatorname{Pr}\left(\|\mathbf{y}-\boldsymbol{\Psi} \mathbf{s}\|^{2}>\left\|\mathbf{y}-\mathbf{\Psi}_{\mathbf{s}_{k}^{\prime}}^{\prime}\right\|^{2}\right) \\
& =\operatorname{Pr}\left(\|\mathbf{n}\|^{2}>\left\|\boldsymbol{\psi}_{l(k)} \sqrt{P_{k}} h_{k}+\mathbf{n}\right\|^{2}\right) \\
& =\mathcal{Q}\left(\sqrt{\frac{\left\|\boldsymbol{\psi}_{l(k)}\right\|^{2} P_{k}\left|h_{k}\right|^{2}}{2 N_{0}}}\right)=\mathcal{Q}\left(\sqrt{\frac{P_{k}\left|h_{k}\right|^{2}}{2 N_{0}}}\right) .
\end{aligned}
$$

The probability in (29) is to be seen as a lower-bound, because $\left\{h_{k}\right\}$ (i.e., the CSI) is to be known, which has to be estimated in a CS-based MUD.

\footnotetext{
${ }^{3}$ In general, for ML detection, an exhaustive search is used. In this case, the complexity grows exponentially with $L$, which makes the use of ML detection impractical. However, we consider ML detection to see achievable performance.
} 
As mentioned earlier, since we only consider the events of MD with only one user not detected, using the union bound, the probability of MD event (per used preamble) becomes

$$
P_{\mathrm{MD}} \leq \frac{1}{K} \sum_{k=1}^{K} P_{k}^{\prime}
$$

For FA events, consider a virtual (active) user, say user $K+$ $i, i \in\{1, \ldots, L-K\}$, that sends preamble $\boldsymbol{\psi}_{l(K+i)}$, where $l(K+i) \in \mathcal{S}^{c}$. For each virtual user, consider a $(K+1)$-sparse vector, denoted by $\mathbf{s}_{K+i}^{\prime \prime}$, that satisfies

$$
\left[\mathbf{s}_{K+i}^{\prime \prime}-\mathbf{s}\right]_{l}= \begin{cases}\sqrt{P_{K+i}} h_{K+i}, & \text { if } l=l(K+i) \\ 0, & \text { o.w. }\end{cases}
$$

Here, $h_{K+i}$ is the channel coefficient for a virtual user (say user $K+i$ ) and $P_{K+i}$ represents its transmit power. Note that although user $K+i$ is not an active user, for the FA event, we consider a hypothesis that this user is incorrectly detected as an active user. To this end, this user's channel coefficient and transmit power are assumed as in (31). Then, the probability that the BS incorrectly chooses $\mathbf{s}_{K+i}^{\prime \prime}$ is

$$
\begin{aligned}
P_{k+i}^{\prime \prime} & =\operatorname{Pr}\left(\|\mathbf{y}-\mathbf{\Psi} \mathbf{s}\|^{2}>\left\|\mathbf{y}-\mathbf{\Psi}_{\mathbf{s}_{K+i}^{\prime \prime}}^{\prime \prime}\right\|^{2}\right) \\
& =\mathcal{Q}\left(\sqrt{\frac{P_{K+i}\left|h_{K+i}\right|^{2}}{2 N_{0}}}\right) .
\end{aligned}
$$

Using the union bound, the probability of FA event (per unused preamble) becomes

$$
P_{\mathrm{FA}} \leq \frac{1}{L-K} \sum_{i=1}^{L-K} P_{k+i}^{\prime \prime} .
$$

Although we do not show the probabilities of MD and FA events with more than 1 user difference, they are much lower than those with 1 user difference in (30) and (33). Note that more detailed analysis is required if the correlation of preambles is high or SNR is low, which could be a further research issue to be studied in the future.

As we can see in (30) and (33), $P_{\mathrm{MD}}$ and $P_{\mathrm{FA}}$ are mainly dependent on the SNR, $\frac{P_{k}\left|h_{k}\right|^{2}}{N_{0}}$. With a power control policy to compensate fading, we may have $P_{k}\left|h_{k}\right|^{2}=P_{\mathrm{rx}}$, where $P_{\mathrm{rx}}$ represents the effective receive power. With a sufficiently high SNR, $P_{\mathrm{MD}}$ and $P_{\mathrm{FA}}$ can be sufficiently low and independent of $K$. This justifies the use of constant error probabilities, $P_{\mathrm{MD}}$ and $P_{\mathrm{FA}}$, in finding the throughput in Section III.

Note that although $P_{\mathrm{MD}}$ and $P_{\mathrm{FA}}$ are sufficiently low, the probability that the transmitted preambles are not correctly detected by MUD (i.e., the error probability) can be high. To see this, let $P_{\mathrm{MD}}=P_{\mathrm{FA}}=\epsilon \ll 1$. Then, assuming that MD and FA events are independent, the error probability becomes

$$
\begin{aligned}
P_{\mathrm{err}} & =\operatorname{Pr}(\hat{\mathcal{S}} \neq \mathcal{S}) \\
& =1-\left(1-P_{\mathrm{FA}}\right)^{L-K}\left(1-P_{\mathrm{FA}}\right)^{K} \\
& \approx 1-e^{-(L-K) \epsilon} e^{-K \epsilon}=1-e^{-L \epsilon},
\end{aligned}
$$

where $\hat{\mathcal{S}}$ represents the estimated support set by MUD. For example, if $\epsilon=0.01$ and $L=310, P_{\text {err }}$ becomes 0.955, which indicates that even if $P_{\text {err }}$ can be high, the probability of individual MD or FA event (per used preamble) can be low.

\section{Simulation Results}

In this section, we present simulation results for the throughput and the average length of Stage 2 of CRA-2, and compare them with the theoretical ones in (18) and (17), respectively. For convenience, let $T=T_{\mathrm{P}}+T_{\mathrm{D}}=N+M$ (in unit symbol duration $^{4}$ ), which is the transmission time required to send one packet with one preamble by one active user. In addition, let $\lambda_{T}=\lambda T$ be the normalized traffic intensity. That is, if $\lambda_{T}=1$, it is expected to have one packet is generated over $T$ on average. The throughput and the traffic intensity will be normalized $^{5}$ in most cases in this section. Note that from (30) and (33), in this section, we assume that $P_{\mathrm{FA}}=P_{\mathrm{MD}}$.

Fig. 4 shows the performance for various normalized traffic intensity $\lambda_{T}$ with $N=31, M=256$ (if binary signaling is used, a data packet can transmit 32 bytes), $\tau=4, L=10 \mathrm{~N}$, and $P_{\mathrm{FA}}=P_{\mathrm{MD}}=0.01$. The throughput curves of the three different schemes, CRA-1, CRA-2, and multichannel ALOHA, are shown in Fig. 4 (a). In Fig. 4 (b), the ratio of the average length of Stage 2, $\bar{D} M$, of CRA-2 to that of CRA-1, NM is shown as a function of $\lambda_{T}$. Clearly, CRA-1 has a higher throughput than multichannel ALOHA by a factor of (nearly) 2, as known in [28]. More importantly, we can see that the throughput of CRA-2 is higher than that of CRA-1 and it can be close 1 thanks to the adaptive length of Stage 2. If the number of active users is sufficiently small, CRA-2 may have a shorter length of Stage 2 than CRA-1 (i.e., $D_{1}+D_{2}+D_{3}<$ $N$ ), which results in a higher throughput (than that of CRA-1). As shown in Fig. 4 (b), we have $\bar{D}<N$ if $\lambda_{T} \leq 1$.

We show performance for various numbers of preambles, $L$, with $\lambda_{T}=1, N=31, M=256, \tau=4$, and $P_{\mathrm{FA}}=P_{\mathrm{MD}}=$ 0.01 in Fig. 5. It is shown that the theoretical results agree with simulation results for a wide range of $L$ (except that $L$ is small ${ }^{6}$ in CRA-2). In general, as the number of preambles, $L$, increases, a higher throughput is achieved in both CRA-1 and CRA-2. However, the throughput becomes saturated once $L$ is sufficiently large. This indicates that it is not necessary to have a large number of preambles. For example, if $L=10 \mathrm{~N}$ might be sufficient to have a reasonable performance in terms of throughput. In Fig. 5 (b), it is also shown that the average length of CRA-2 increases with $L$. If $L \leq 450$, we see that the average length of CRA-2 is shorter than that of CRA-1. Thus, with not too large $L, C R A-2$ can provide a higher throughput and a shorter delay than CRA-1.

In Fig. 6, we set $\lambda$ to $\frac{1}{200}$ and show the performance for various lengths of packet, $M$, with $N=31, L=10 N, \tau=4$, and $P_{\mathrm{FA}}=P_{\mathrm{MD}}=0.01$. It is shown that the throughput increases with $M$ and then decreases in all the random access schemes. Thus, in each scheme, there is a best length of data packet that maximizes the throughput. It is shown in Fig. 6

\footnotetext{
${ }^{4}$ The unit symbol duration is the inverse of the bandwidth if the Nyquis sampling rate is used. All the lengths of sequences (e.g., $N, M, T$, and so on) and feedback duration (e.g., $\tau$ ) are given in unit symbol duration.

${ }^{5}$ The normalized throughput is the average number of successfully recovered packets per $T=N+M$. That is, the normalized throughput is $T \eta_{1}$ and $T \eta_{2}$ for CRA 1 and CRA 2, respectively.

${ }^{6}$ As mentioned earlier, the assumption of A1) is reasonably when $L \gg K$. Thus, if $L$ is small, $\eta_{2}$ in (18) may differ from simulation results.
} 


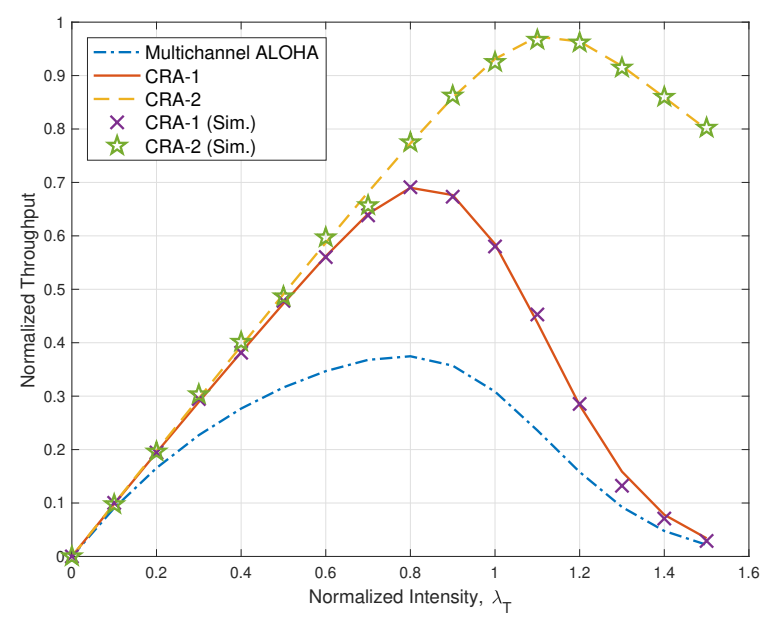

(a)

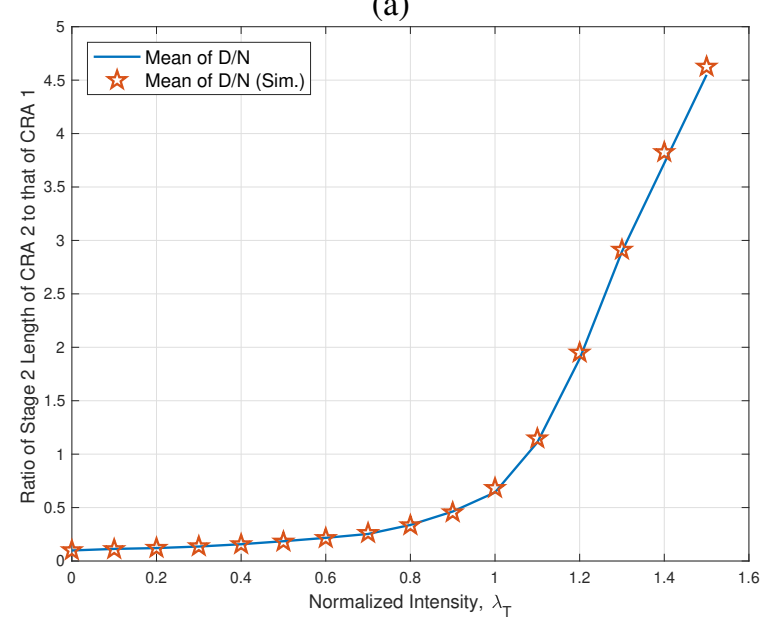

(b)

Fig. 4. Performance for various normalized traffic intensity $\lambda_{T}$ with $N=31$, $M=256, \tau=4, L=10 N$, and $P_{\mathrm{FA}}=P_{\mathrm{MD}}=0.01$ : (a) Normalized throughput, $T \eta_{i}, i \in\{1,2, \mathrm{ma}\}$; (b) the ratio of the average length of Stage 2, $\bar{D} M$, of CRA-2 to that of CRA-1, NM.

(b) that $M$ should not be too long to avoid a long delay and a low throughput in CRA-2.

The impact of the probabilities of MD and FA on the performance is shown in Fig. 7 with $\lambda_{T}=1, N=31$, $L=10 N, \tau=4$, and $M=256$. It is shown that CRA2 has a more throughput degradation than other schemes as $P_{\mathrm{MD}}=P_{\mathrm{FA}}$ increases thanks to slots generated by FA events, (i.e., $D_{3}$ slots). However, as long as $P_{\mathrm{FA}}=P_{\mathrm{MD}}$ is sufficiently low, CRA-2 outperforms the others. In addition, as shown in Fig. 7 (b), the average length of Stage 2 increases with $P_{\mathrm{FA}}=P_{\mathrm{MD}}$. Thus, to avoid a long delay, it is necessary to keep $P_{\mathrm{FA}}=P_{\mathrm{MD}}$ low.

\section{CONCLUDING REMARKS}

In a number of IoT applications, each active IoT device or sensor may have a short data packet (of a few ten bytes) and one short message delivery would be required at each access to uplink. Thus, we considered CRA-based random access schemes when active devices or sensors have one data packet of the same length in this paper. Based on a simplified

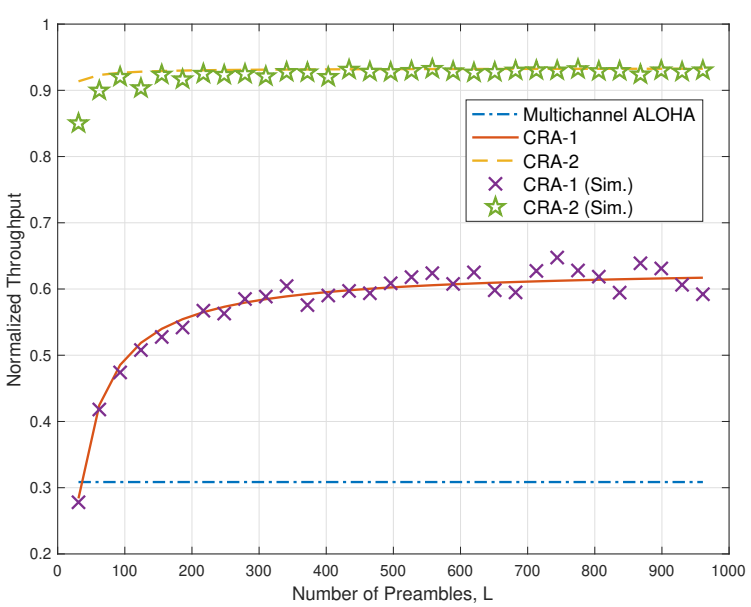

(a)

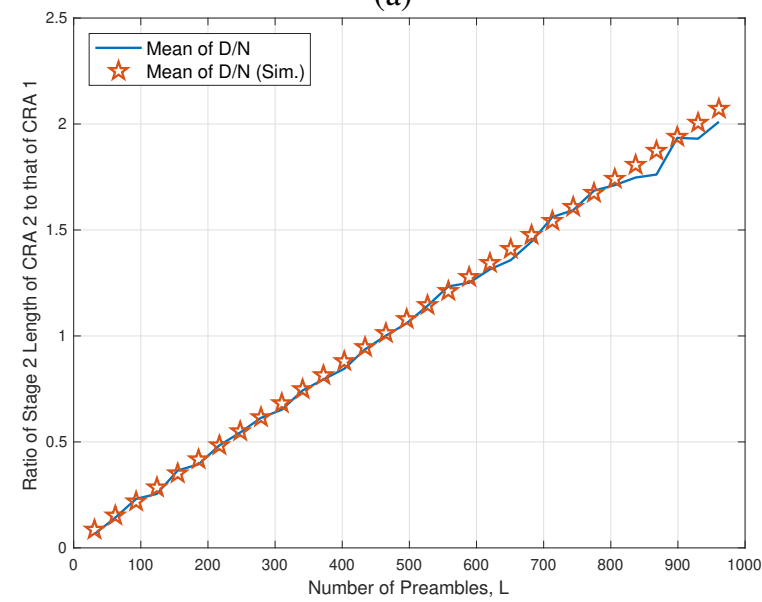

(b)

Fig. 5. Performance for various numbers of preambles, $L$, with $\lambda_{T}=1$, $N=31, M=256, \tau=4$, and $P_{\mathrm{FA}}=P_{\mathrm{MD}}=0.01$ : (a) Normalized throughput, $T \eta_{i}, i \in\{1,2, \mathrm{ma}\}$; (b) the ratio of the average length of Stage 2, $\bar{D} M$, of CRA-2 to that of CRA-1, NM.

handshaking process, a CRA-based random access scheme was studied and analyzed together with a conventional CRA-based random access (which is a grant-free approach). Since the length of payload can be adaptively decided depending on the number of active users, it was shown that the CRA-based random access scheme with simplified handshaking process can outperform others. We derived the throughput expressions and showed that they agree with simulation results. Thus, the throughput expressions could be used to design a CRAbased random access system with IoT devices that have short messages to be delivered in MTC.

While we did not consider massive MIMO in this paper, it is possible to generalize the approach with massive MIMO based random access [12] [13] [14] [16] [17] in order to increase the number of users to be supported, which might be a further research topic.

\section{APPENDIX A \\ PROOF OF LEMMA 1}

From (6), it can be shown that $B=\sum_{l=1}^{L} U_{l}+W_{l}$, while $B_{1}=\sum_{l=1}^{L} U_{l}$. Then, under the assumption of $\mathbf{A} \mathbf{1}, B$ and $B_{1}$ 


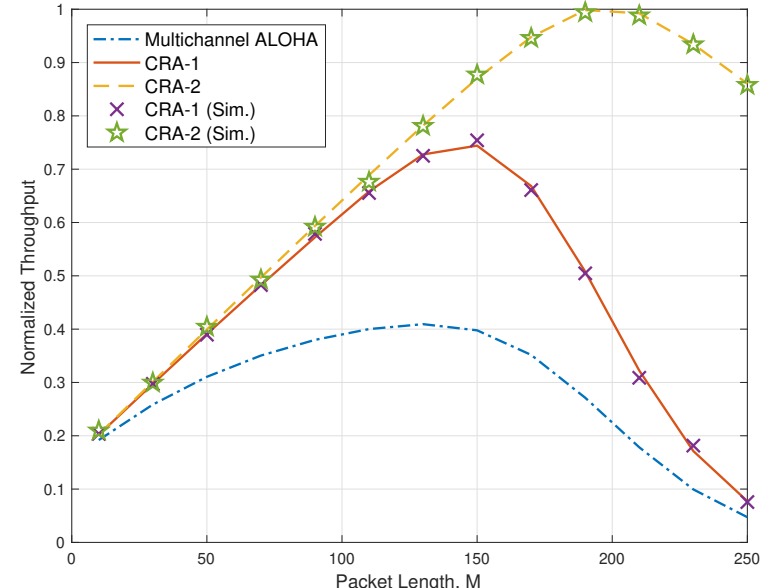

(a)

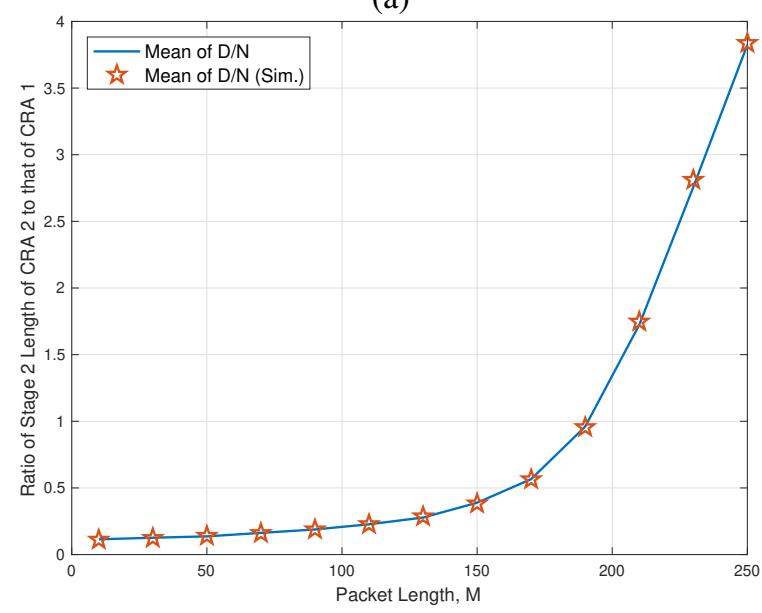

(b)

Fig. 6. Performance for various lengths of packets, $M$, with $\lambda=\frac{1}{200}$, $N=31, L=10 N, \tau=4$, and $P_{\mathrm{FA}}=P_{\mathrm{MD}}=0.01$ : (a) Normalized throughput, $T \eta_{i}, i \in\{1,2, \mathrm{ma}\}$; (b) the ratio of the average length of Stage 2, $\bar{D} M$, of CRA-2 to that of CRA-1, NM.

become binomial random variables as follows:

$$
\begin{gathered}
\operatorname{Pr}\left(B_{1}=b_{1}\right)=\left(\begin{array}{c}
L \\
b_{1}
\end{array}\right) \alpha_{1}^{b_{1}}\left(1-\alpha_{1}\right)^{L-b_{1}} \\
\operatorname{Pr}(B=b)=\left(\begin{array}{c}
L \\
b
\end{array}\right)\left(1-\alpha_{2}\right)^{b} \alpha_{2}^{L-b} .
\end{gathered}
$$

Since $\mathbb{E}\left[D_{1} \mid B_{1}\right]=\left(1-P_{\mathrm{MD}}\right) B_{1}$, from (35) we have

$$
\mathbb{E}\left[D_{1} \mid K\right]=\left(1-P_{\mathrm{MD}}\right) \mathbb{E}\left[B_{1} \mid K\right]=\left(1-P_{\mathrm{MD}}\right) L \alpha_{1} .
$$

Using the same approach, we have

$$
\begin{aligned}
\mathbb{E}\left[D_{2} \mid K\right] & =\left(1-P_{\mathrm{MD}}\right) \mathbb{E}\left[B_{2} \mid K\right] \\
& =\left(1-P_{\mathrm{MD}}\right) \mathbb{E}\left[B-B_{1} \mid K\right] \\
& =\left(1-P_{\mathrm{MD}}\right) L\left(1-\alpha_{1}-\alpha_{2}\right) .
\end{aligned}
$$

and

$$
\mathbb{E}\left[D_{3} \mid K\right]=P_{\mathrm{FA}} \mathbb{E}[L-B \mid K]=P_{\mathrm{FA}} L\left(1-\alpha_{2}\right) .
$$

From (36) - (37), we have (10), which completes the proof.

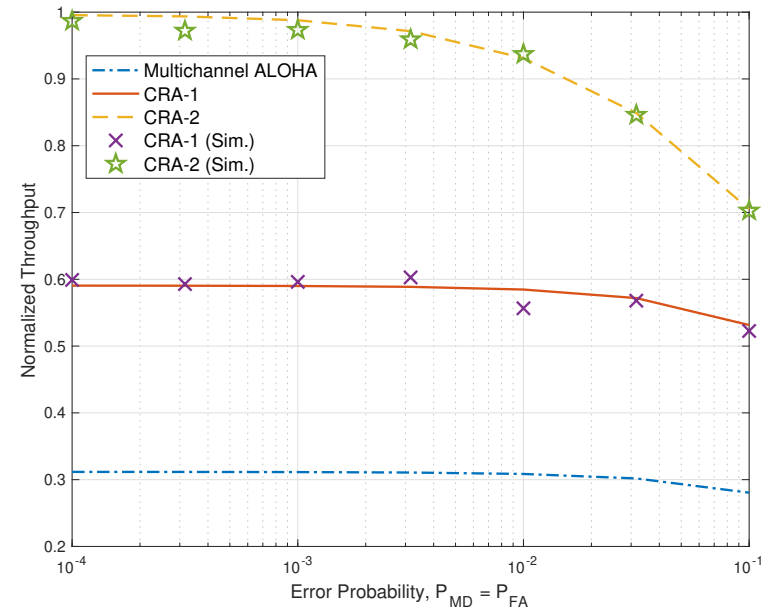

(a)

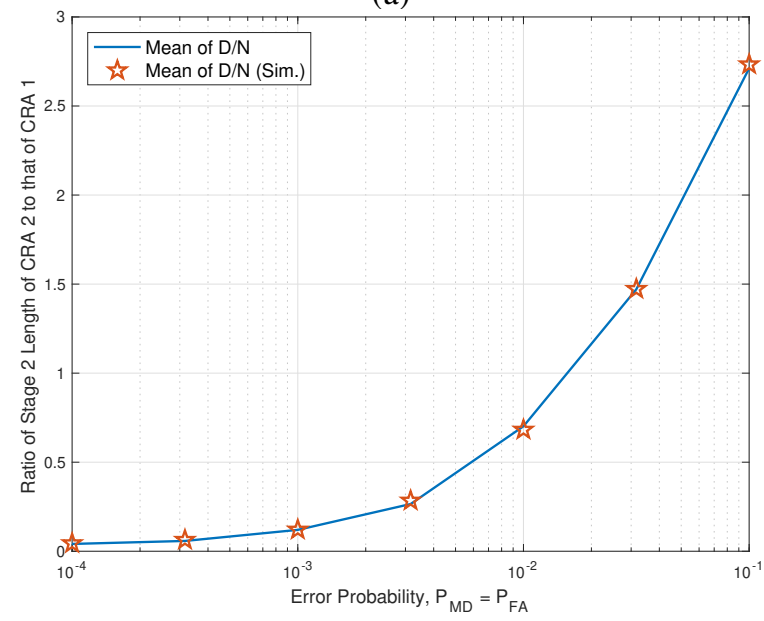

(b)

Fig. 7. Performance for various probabilities of MD and FA with $\lambda_{T}=1$, $N=31, L=10 N, \tau=4$, and $M=256$ : (a) Normalized throughput, $T \eta_{i}, i \in\{1,2, \mathrm{ma}\}$; (b) the ratio of the average length of Stage $2, \bar{D} M$, of CRA-2 to that of CRA-1, NM.

\section{APPENDIX B}

PROOF OF LEMMA 2

From (11), it follows that

$$
\bar{Y}=\tilde{T}_{\mathrm{P}}+T_{\mathrm{D}} \bar{D}
$$

where $\bar{D}=\mathbb{E}[D]=\mathbb{E}[\mathbb{E}[D \mid K]]$. Here, the first expectation is carried out over $K$ that has the distribution in (13). Using (13) and (10), after some manipulations, it can be shown that

$$
\bar{D}=L\left(\left(1-P_{\mathrm{MD}}\right)-e^{-\frac{\beta_{2}}{L}}\left(1-P_{\mathrm{MD}}-P_{\mathrm{FA}}\right)\right) .
$$

In addition, from (39), we have

$$
\beta_{2}=\lambda\left(\tilde{T}_{\mathrm{P}}+T_{\mathrm{D}} \bar{D}\right) .
$$

Substituting (40) into (41), we have

$$
\frac{\beta_{2}}{L}=c_{1}-c_{2} e^{\frac{-\beta_{2}}{L}} \text {. }
$$

Let $t=c_{1}-\frac{\beta_{2}}{L}$. Then, (42) is re-written as $t=c_{2} e^{t} e^{-c_{1}}$ or

$$
-t e^{-t}=-c_{2} e^{-c_{1}},
$$


which implies that (by the definition of the Lambert W function)

$$
-t=\mathbb{W}\left(-c_{2} e^{-c_{1}}\right) .
$$

From this, we can obtain the expression for $\beta_{2}$ in (14). Substituting (42) and (14) into (40), we have (15).

\section{REFERENCES}

[1] J. Gubbi, R. Buyya, S. Marusic, and M. Palaniswami, "Internet of Things (IoT): A vision, architectural elements, and future directions," Future Gener. Comput. Syst., vol. 29, pp. 1645-1660, Sept. 2013.

[2] J. Kim, J. Yun, S. Choi, D. N. Seed, G. Lu, M. Bauer, A. AlHezmi, K. Campowsky, and J. Song, "Standard-based IoT platforms interworking: implementation, experiences, and lessons learned," IEEE Communications Magazine, vol. 54, pp. 48-54, July 2016.

[3] N. Mangalvedhe, R. Ratasuk, and A. Ghosh, "NB-IoT deployment study for low power wide area cellular IoT," in 2016 IEEE 27th Annual International Symposium on Personal, Indoor, and Mobile Radio Communications (PIMRC), pp. 1-6, Sep. 2016.

[4] 3GPP TS 36.321 V13.2.0, Evolved Universal Terrestrial Radio Access (E-UTRA); Medium Access Control (MAC) protocol specification, June 2016.

[5] 3GPP TR 37.868 V11.0, Study on RAN improvments for machine-type communications, October 2011.

[6] H. Shariatmadari, R. Ratasuk, S. Iraji, A. Laya, T. Taleb, R. Jntti, and A. Ghosh, "Machine-type communications: current status and future perspectives toward 5G systems," IEEE Communications Magazine, vol. 53, pp. 10-17, September 2015.

[7] O. Galinina, A. Turlikov, S. Andreev, and Y. Koucheryavy, "Stabilizing multi-channel slotted ALOHA for machine-type communications," in Proc. IEEE ISIT, pp. 2119-2123, July 2013.

[8] C. H. Chang and R. Y. Chang, "Design and analysis of multichannel slotted ALOHA for machine-to-machine communication," in Proc. IEEE GLOBECOM, pp. 1-6, Dec 2015.

[9] J. Choi, "On the adaptive determination of the number of preambles in RACH for MTC," IEEE Communications Letters, vol. 20, pp. 13851388, July 2016.

[10] C. Bockelmann, N. Pratas, H. Nikopour, K. Au, T. Svensson, C. Stefanovic, P. Popovski, and A. Dekorsy, "Massive machine-type communications in 5G: physical and MAC-layer solutions," IEEE Communications Magazine, vol. 54, pp. 59-65, September 2016.

[11] T. L. Marzetta, "Noncooperative cellular wireless with unlimited numbers of base station antennas," IEEE Trans. Wireless Commun., vol. 9, pp. 3590-3600, Nov. 2010.

[12] E. de Carvalho, E. Bjrnson, J. H. Srensen, E. G. Larsson, and P. Popovski, "Random pilot and data access in massive MIMO for machine-type communications," IEEE Trans. Wireless Communications, vol. 16, pp. 7703-7717, Dec 2017.

[13] K. Senel and E. G. Larsson, "Device activity and embedded information bit detection using AMP in massive MIMO," in 2017 IEEE Globecom Workshops (GC Wkshps), pp. 1-6, Dec 2017.

[14] L. Liu, E. G. Larsson, W. Yu, P. Popovski, C. Stefanovic, and E. de Carvalho, "Sparse signal processing for grant-free massive connectivity: A future paradigm for random access protocols in the Internet of Things," IEEE Signal Processing Magazine, vol. 35, pp. 88-99, Sept 2018.

[15] L. Liu and W. Yu, "Massive connectivity with massive MIMO-part I. Device activity detection and channel estimation," IEEE Trans. Signal Processing, vol. 66, pp. 2933-2946, June 2018.

[16] F. Jiang, J. Chen, A. L. Swindlehurst, and J. A. Lpez-Salcedo, "Massive MIMO for wireless sensing with a coherent multiple access channel," IEEE Trans. Signal Processing, vol. 63, pp. 3005-3017, June 2015.

[17] D. Ciuonzo, P. S. Rossi, and S. Dey, "Massive MIMO channel-aware decision fusion," IEEE Trans. Signal Processing, vol. 63, pp. 604-619, Feb 2015.

[18] E. Bjrnson, J. Hoydis, and L. Sanguinetti, "Massive MIMO has unlimited capacity," IEEE Trans. Wireless Communications, vol. 17, pp. 574-590, Jan 2018.

[19] H. Zhu and G. Giannakis, "Exploiting sparse user activity in multiuser detection," IEEE Trans. Communications, vol. 59, pp. 454-465, February 2011.

[20] L. Applebaum, W. U. Bajwa, M. F. Duarte, and R. Calderbank, "Asynchronous code-division random access using convex optimization," Physical Communication, vol. 5, no. 2, pp. 129-147, 2012.
[21] H. F. Schepker, C. Bockelmann, and A. Dekorsy, "Exploiting sparsity in channel and data estimation for sporadic multi-user communication," in Proc. ISWCS 2013, pp. 1-5, Aug 2013.

[22] J. Choi, "Two-stage multiple access for many devices of unique identifications over frequency-selective fading channels," IEEE Internet of Things J., vol. 4, pp. 162-171, Feb 2017.

[23] A. T. Abebe and C. G. Kang, "Comprehensive grant-free random access for massive low latency communication," in 2017 IEEE International Conference on Communications (ICC), pp. 1-6, May 2017.

[24] G. Wunder, P. Jung, and C. Wang, "Compressive random access for post-LTE systems," in Proc. IEEE ICC, pp. 539-544, June 2014.

[25] D. Donoho, "Compressed sensing," IEEE Trans. Information Theory, vol. 52, pp. 1289-1306, April 2006.

[26] E. Candes, J. Romberg, and T. Tao, "Robust uncertainty principles: exact signal reconstruction from highly incomplete frequency information," IEEE Trans. Information Theory, vol. 52, pp. 489-509, Feb 2006.

[27] H. Seo, J. Hong, and W. Choi, "Low latency random access for sporadic MTC devices in internet of things," IEEE Internet of Things J., vol. 6, pp. 5108-5118, June 2019.

[28] J. Choi, "Stability and throughput of random access with CS-based MUD for MTC," IEEE Trans. Vehicular Technology, vol. 67, pp. 2607-2616, March 2018.

[29] N. Naik, "Choice of effective messaging protocols for IoT systems: MQTT, CoAP, AMQP and HTTP," in 2017 IEEE International Systems Engineering Symposium (ISSE), pp. 1-7, Oct 2017.

[30] S. Verdu, Multiuser Detection. Cambridge University Press, 1998.

[31] J. Chen and X. Huo, "Theoretical results on sparse representations of multiple-measurement vectors," IEEE Trans. Signal Processing, vol. 54, pp. 4634-4643, Dec 2006.

[32] M. E. Davies and Y. C. Eldar, "Rank awareness in joint sparse recovery," IEEE Trans. Information Theory, vol. 58, pp. 1135-1146, Feb 2012.

[33] D. Donoho and M. Elad, "Optimally sparse representation in general (nonorthogonal) dictionaries via L1 minimization," in Proc. Natl. Acad. Sci. 2003, pp. 2197-2202, March 2003.

[34] A. M. Bruckstein, D. L. Donoho, and M. Elad, "From sparse solutions of systems of equations to sparse modeling of signals and images," SIAM Review, vol. 51, no. 1, pp. 34-81, 2009.

[35] J. Choi, Optimal Combining and Detection. Cambridge University Press, 2010.

[36] Y.-J. Choi, S. Park, and S. Bahk, "Multichannel random access in OFDMA wireless networks," IEEE J. Selected Areas in Communications, vol. 24, pp. 603-613, March 2006.

[37] F. Kelly and E. Yudovina, Stochastic Networks. Cambridge University Press, 2014.

[38] Y. C. Eldar and G. Kutyniok, Compressed Sensing: Theory and Applications. Cambridge University Press, 2012.

[39] R. Tibshirani, "Regression shrinkage and selection via the lasso," Journal of the Royal Statistical Society. Series B (Methodological), vol. 58 , no. 1, pp. 267-288, 1996. 